\title{
Impact of the COVID-19 Pandemic on Trauma and Orthopaedic Service in the Republic of Ireland
}

\author{
Authors: \\ Hany Elbardesy \\ Department of Trauma and Orthopaedics, Cork University Hospital, Cork, Ireland \\ *Correspondence to elbardecy@hotmail.com \\ Disclosure: \\ The author has declared no conflicts of interest. \\ Received: \\ 27.04.20 \\ Accepted: \\ 08.06 .20 \\ Keywords: \\ COVID-19, Ireland, trauma, surgeries. \\ Citation: \\ EMJ Microbiol Infect Dis. 2020;DOI/10.33590/emjmicrobiolinfectdis/20-00116
}

\section{Abstract}

Background: The novel coronavirus disease-2019 (COVID-19) has been identified as the cause of a rapidly spreading respiratory illness that is thought to have originated from Wuhan, China in early December 2019. Since then, the free movement of people has decreased, which has thus reduced the number of trauma-related casualties. The Irish governments initiated strict social distancing measures in response to the COVID-19 pandemic in late March 2020. It remains challenging to quantify the impact this had on reducing the spread of the virus. The viral outbreak has led to significant changes in the lifestyle of Irish citizens. The aim of this study was to examine the impact of the pandemic on activity, related to emergencies in trauma and orthopaedics departments.

Methods: Patients admitted to the Trauma and Orthopaedic Department at Cork University Hospital (CUH), Cork, Ireland and South Infirmary Victoria University Hospital (SIVUH), Cork, Ireland between the $1^{\text {st }}$ of March and the $15^{\text {th }}$ of April 2020 were documented and compared to the patient admissions from the same time period in 2019.

Results: The total numbers of lower limb trauma cases dropped by $20.44 \%$ from 137 (females: 87; males: 50 ) to 109 (females: 74; male: 35$),(p=0.72)$. Of note, there was a $30.00 \%$ reduction in the number of male cases (from 50 to $35 ; \mathrm{p}<0.05$ ), in comparison to the $14.94 \%$ reduction observed in the female population (from 87 to $74 ; \mathrm{p}<0.05$ ). The total number of upper limb trauma cases increased by $0.14 \%$ from 90 (female: 44; men: 46) to 94 (female: 54; male: 40; $p=0.14$ ). The increase reported was only applicable to the female population; the number of male cases of upper limb trauma slightly decreased from 46 to 40 ( $p<0.005)$.

Conclusion: The COVID-19 crisis has led to a decrease in the total numbers of lower limb trauma surgeries but an increase in the number of upper limb fractures. The main reduction was amongst the male patients, while the number of female cases increased.

\section{INTRODUCTION}

The COVID-19 pandemic is a catastrophic global healthcare crisis that is believed to have originated from Wuhan, China in late 2019,2 and has since spread at an exponential rate worldwide. $^{3}$ Healthcare systems have been stretched physically, financially, and mentally in an effort to curtail this crisis, with damage- 
limitation at the forefront of these measures. The COVID-19 pandemic was characterised by different responses from authorities around the world. Some initiated early social distancing measures and mandatory shutdown of all nonessential outdoor activities, while others depended on thorough test and trace strategies. ${ }^{4}$ Amongst this heterogeneity, it is difficult to quantify the effect of the singular actions and what may have occurred in the absence of such efforts. These efforts manifested in many different ways, from redeploying healthcare staff5 to building new temporary hospitals in a matter of days/weeks. ${ }^{6}$ Most countries have introduced compulsory partial or total lockdown. In Ireland, the government instigated total lockdown to try to slow the spread of COVID-19 in March 2020.7 The lockdown included: asking people to stay at home, closure of schools and universities and nonessential facilities (excluding supermarkets and health care utilities), rationing of supplies, requisition of facilities, deferral of international travel, limitation of internal journeys, and, primarily, reprioritisation of healthcare services. ${ }^{8}$

From this lockdown initiation date, the number of accidents related to outdoor activities were expected to decline. The Royal College of Surgeons of Ireland (RCSI) published new guidance for surgical prioritisation. It classified surgeries into four categories according to their level of urgency: priority Level 1a refers to an emergency that requires surgery within 24 hours; priority Level 1b was classified as an urgent operation needed within 72 hours; priority Level 2 was surgery that can be deferred for up to 4 weeks; priority Level 3 surgery could be delayed for up to 3 months; and priority Level 4 refers to surgery that could be delayed for $>3$ months. ${ }^{9}$

Consequently, all elective cases were postponed, limiting the demand on healthcare resources and keeping hospital beds available, if needed, for emergency admissions. Other countries such as Singapore, Australia, and New Zealand employed different approaches with the same underlying principles that were based on clinical urgency, patient and healthcare worker protection, and conservation of healthcare resources. ${ }^{5,10}$ Australia implemented a similar but slightly less strict nationwide response on the $29^{\text {th }}$ March 2020." The relationship between this COVID-19 pandemic and orthopaedic surgery appears disparate when compared to other medical specialities such as infectious diseases, emergency medicine, and internal medicine. However, as part of the larger healthcare ecosystem, orthopaedic surgeons also have a role in this pandemic. This study was conducted to measure the effect this crisis was having on the trauma and orthopaedic (T\&O) departments in the Republic of Ireland, to conclude whether the departments' response was effective.

\section{PATIENTS AND METHODS}

The number of patients admitted to the T\&O department at Cork University Hospital (CUH), Cork, Ireland and the South Infirmary Victoria University Hospital (SIVUH), Cork, Ireland between the $1^{\text {st }}$ of March 2020 and the $15^{\text {th }}$ of April 2020 were recorded and this number was compared to the patient data from the same time period in 2019. All consecutive patients under consultation for T\&O and requiring urgent care at $\mathrm{CUH}$ and the SIVUH during the lockdown period (case group) and the equivalent period in 2019 (control group) were included.

$\mathrm{CUH}$ is a Level 1 trauma centre and it is the only hospital in Cork that manages T\&O. The city of Cork covers a total surface area of 820 $\mathrm{km}^{2}$ in South Ireland. The population density is approximately 3,300 individuals per $\mathrm{km}^{2}$ in the city, and this density drops to approximately 480 per $\mathrm{km}^{2}$ when looking at the metropolitan area. The current population is 417,211 , with 206,953 males and 210,258 females. ${ }^{2}$ Typically, prepandemic, all trauma surgeries were performed in two operating rooms (OR) at CUH daily, except for weekends and public holidays when only one OR was available. SIVUH was used solely for elective cases. Since the advent of the crisis, CUH has only one dedicated OR for T\&O surgery that is kept exclusively for elderly patients who are medically unwell, which mostly includes patients with hip fractures. The reasoning behind this approach was based on the literature that documented that older patients with medical comorbidities were more adversely affected by COVID-19 infection owing to their low functional reserves and weakened immune systems. ${ }^{13-15}$

The inclusion period for the case group corresponds to the confinement period in Ireland ( $1^{\text {st }}$ March 2020 to $15^{\text {th }}$ April 2020). The inclusion 
period for the control group corresponds to the same time period in 2019. All patients with prosthetic joint infections which were not related to outdoors activities were excluded, as well as all spine trauma cases, as they were all either treated by the neurosurgery team or transferred to the local spinal unit in Dublin, Ireland. ${ }^{16}$ The type of treatment (conservative or surgical) and the hospitalisation procedures (outpatient or conventional hospitalisation) were also reported.

\section{Data Collection}

All demographic data, medical history, and clinical and radiological data at presentation was collected. All trauma injuries requiring urgent care were divided into two categories: upper and lower limb. The anatomical location of the injury was also recorded so that it could be compared.

\section{Statistical Analyses}

Continuous variables are presented as mean with standard deviation; categorical variables are presented as count (\%). Independent twosample t-test and Chi-square test were used, as appropriate, to compare groups. Statistical significance was defined as $\mathrm{p}<0.05$.

\section{RESULTS}

\section{Lower Limb Trauma}

The total number of lower limb trauma cases dropped by $20.44 \%$ from 137 (females: 87; male: 50 ) to 109 (females: 74; male: $35 ; p=0.72$ ). Of note, there was a $30.00 \%$ reduction in the number of male cases (from 50 to 35; $\mathrm{p}<0.05$ ), in comparison to the $14.94 \%$ reduction observed in the female population (from 87 to $74 ; \mathrm{p}<0.05$ ).

\section{Upper Limb Trauma}

The total number of upper limb trauma cases increased by $0.14 \%$ from 90 (female: 44; men: 46 ) to 94 (female: 54; male: 40; $p=0.14$ ). The increase reported was only applicable to the female population; the number of male cases of upper limb trauma slightly decreased from 46 to $40(p<0.005)$.

A breakdown of operative numbers and population data can be found in Tables 1 and 2 and Figures 1 and 2.

\section{DISCUSSION}

As the current health crisis was unprecedented, no one was able to predict the consequences of such a lockdown on hand and upper extremity emergencies. Many studies have proposed guidelines to adapt the organisation of care services to cope with this outbreak, but to our knowledge, this review is the first to analyse the direct impact of this pandemic and the response to trauma emergency aetiologies and management. Some authors have highlighted a definite change in the aetiologies of injuries, which feature almost no work-related injuries and less road traffic and leisure accidents but a significant increase in domestic accidents..$^{17}$ Many patients occupied themselves with gardening, cooking, and handiwork. To some extent, it is possible to compare these results to those observed during special events such as the annual Super Bowl in the USA. People are used to staying at home, watching TV and cooking, temporarily living a very short experimental lockdown. ${ }^{18}$

Additionally, the lockdown has had many psychological impacts on the population, with an increase in domestic violence and anger accidents. This extraordinary social situation has led to an increase in the level of stress and anxiety of entire populations, starting with the patients who had to consult with the hospital. The mental health effects of the COVID-19 pandemic might be profound..$^{19}$ Interventions to address the psychological and social long-term repercussions of the pandemic are required. ${ }^{20}$ Moreover, these results confirm a considerable decrease in the rate of lower limb trauma compared to the reference period in 2019, as well as many other emergencies. For example, in some countries, the number of acute stroke admissions dropped by $50 \% .{ }^{21}$ This reduction in activity has enabled the relocation of surgeon teams to other sectors. All over the world, different actions were put in place in response to the COVID-19 pandemic by orthopaedic surgeons. ${ }^{22-24}$

In this study, formalised patient care was created at the hospital level. Upon arrival in the emergency room, suspected or confirmed COVID-19 patients followed a circuit sealed from COVID-19 negative patients. ${ }^{25}$ 
Table 1: Comparison between the numbers of patients undergoing surgery at Cork University Hospital, Cork, Ireland and South Infirmary Victoria University Hospital, Cork, Ireland in March-April 2019 and 2020.

\begin{tabular}{|l|l|l|l|l|}
\hline Number of surgeries & Control group & Case group & Percentage change & p value \\
\hline Lower limb & 137 & 109 & $-20.44 \%$ & 0.72 \\
\hline Upper limb & 90 & 94 & $+4.44 \%$ & 0.14 \\
\hline
\end{tabular}

Table 2: Patient sex.

\begin{tabular}{|l|l|l|l|}
\hline \multirow{2}{*}{} & \multicolumn{2}{|l|}{ Sex (female/male) } & \multirow{2}{*}{ p value } \\
\cline { 2 - 4 } & Control group & Case group & \\
\hline Lower limb & $87 / 50$ & $74 / 35$ & 0.001 \\
\hline Upper limb & $44 / 46$ & $54 / 40$ & 0.007 \\
\hline
\end{tabular}

\section{Patient's sex}

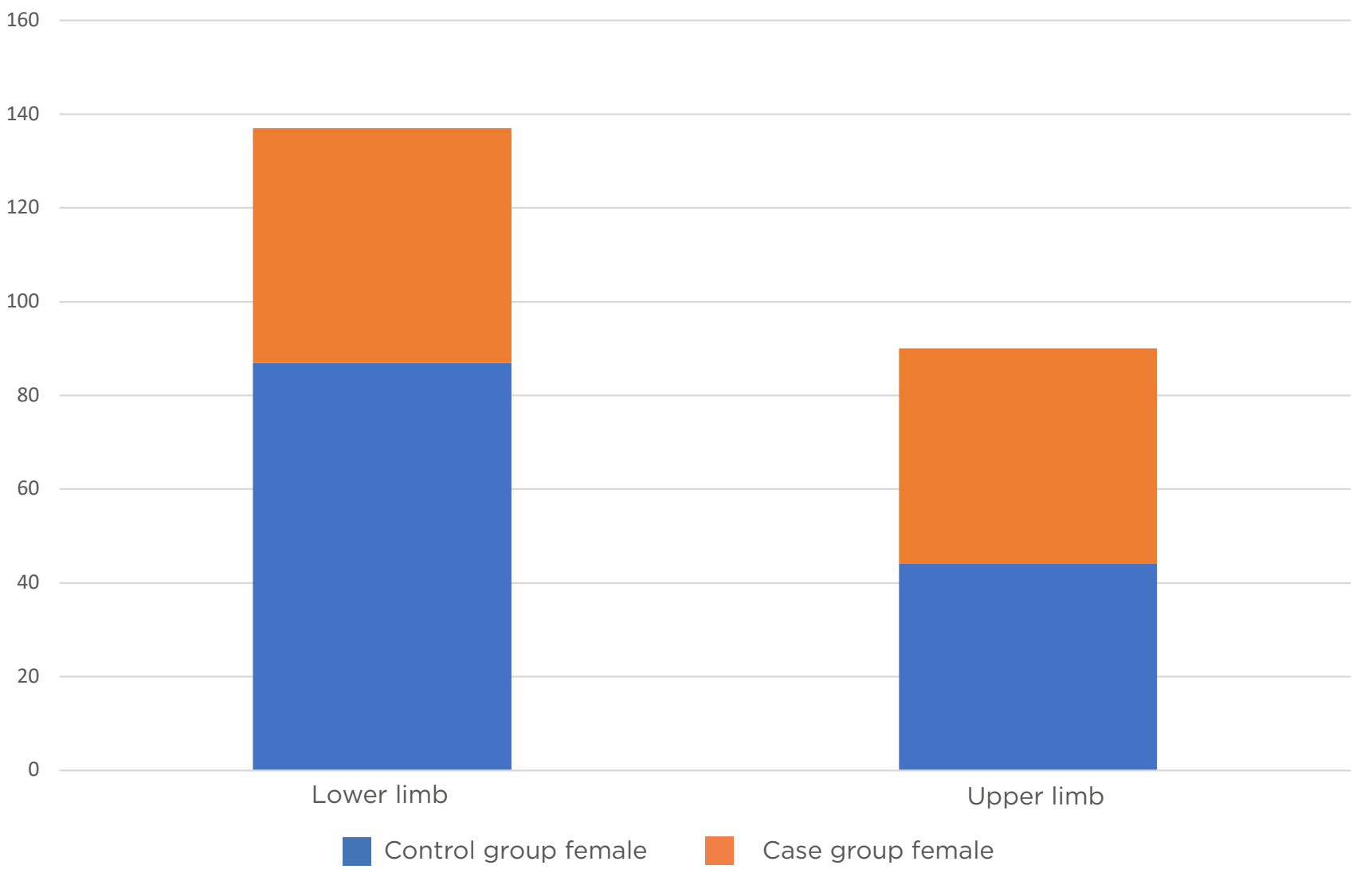

Figure 1: The incidence of trauma according to the patient's sex. 


\section{Comparison between the number of patients undergoing surgery at $\mathrm{CUH}$ and SIVUH}

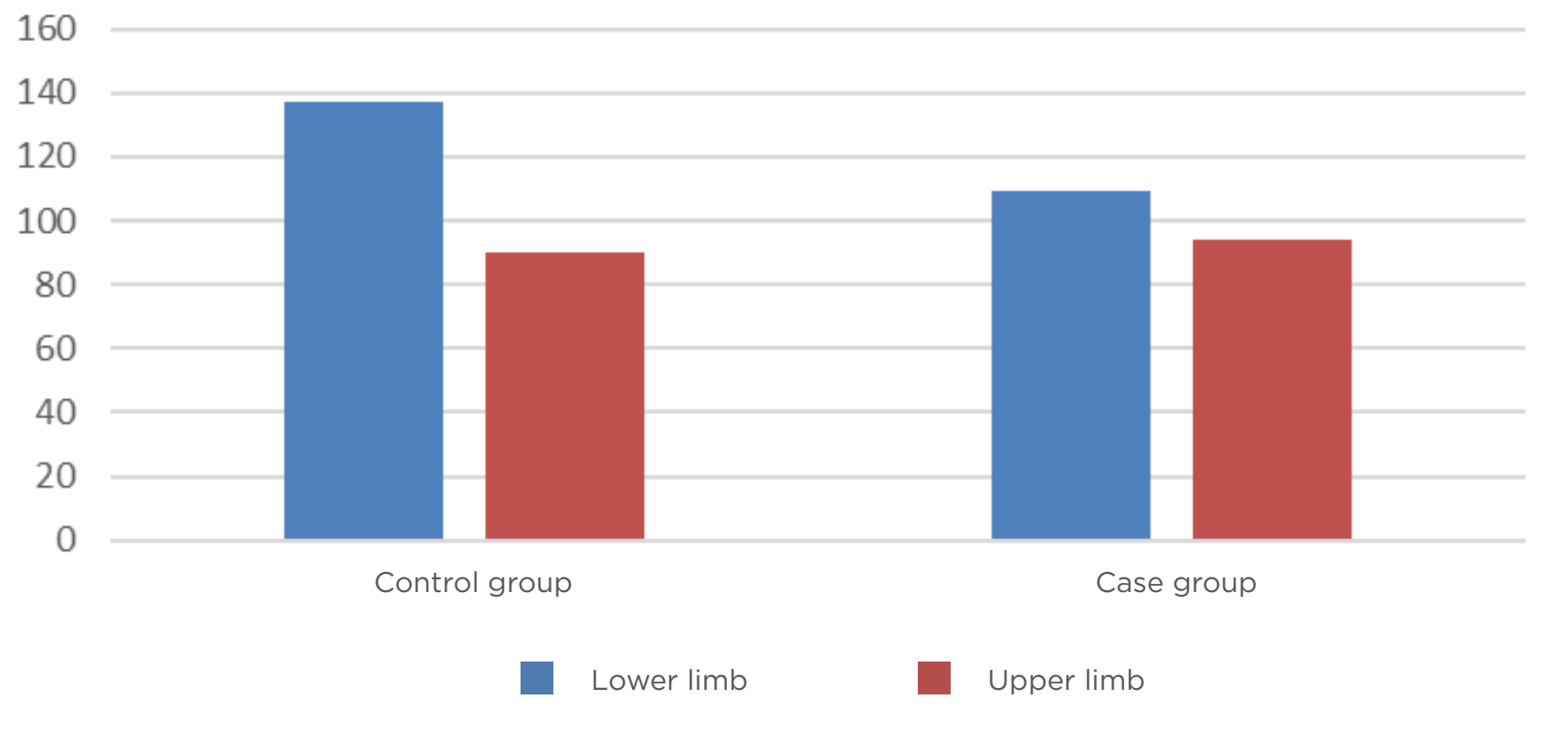

Figure 2: Comparison between the case group and a control group.

CUH: Cork University Hospital; SIVUH: South Infirmary Victoria University Hospital.

With the limitation of log-on to operating rooms and anaesthetic backing, indications for conservative treatment were driven to the maximum. Anaesthetic procedures like wide-awake local anaesthesia no tourniquet (WALANT) have been proposed to continue the care of patients with trauma injuries without an anaesthetic team and crucially without generating aerosolised particles which could put healthcare workers lives' at higher risk..$^{17}$ Indeed, nursing staff and orthopaedic and anaesthetic teams are known to have been infected with COVID $19 .{ }^{26}$ In this study, protection rules to adequately protect health surgeons were applied. ${ }^{27,28}$ Unfortunately, three patients with confirmed COVID-19 operated in this study cohort were unable to benefit from this technique. Despite the risks involved and the organisational constraints, staff treated all patients optimally without reducing the level of quality care.

This pandemic happened in the digital age and has hugely changed working habits. In particular, new services such as telehealth, virtual visits, and online tools for postoperative rehabilitation have been developed. ${ }^{29,30}$ These technologies will accompany virtual and enhanced reality training and digitalisation of meetings in the healthcare system in the future.

In the author's department, the hospital authorities increased the number of inpatient beds from 66 to 90 and the number of operating rooms from two to three. Additionally, all elective surgeries were cancelled to preserve valuable equipment, nurses, and essential staff resources to treat the virus. ${ }^{31}$ The COVID-19 crisis impacted the numbers of T\&O operations performed in a Level 1 major trauma centre in the South of Ireland, but not to a great extent; only a $10.15 \%$ decline was observed.

Because individual sporting activities like running and cycling were not prohibited, the numbers of lower limb trauma actually decreased by $20.44 \%$, possibly because these fracture types require high-impact trauma, ${ }^{32}$ accounted for mostly by road traffic accidents. ${ }^{8}$ 
The number of females requiring T\&O Surgery between the two time periods observed was increased, and any reduction in overall numbers was accounted for by the fall in the number of male patients only; down from 96 to 75 , illustrating a $21.87 \%$ decrease.

\section{CONCLUSION}

The COVID-19 crisis has led to a decrease in the total numbers of lower limb trauma surgeries. However, the upper limb fractures all increased. The main reduction was among the male patients, whilst the number of females presenting with upper limb fractures increased.

\section{STUDY LIMITATION}

The author acknowledges that a limitation of this study is the collection of data from one centre only. Multicentre studies with larger cohorts are warranted to add to the growing body of literature documenting the impact of COVID-19 on T\&O surgery not only locally, but internationally.

\section{References}

1. Zhu $\mathrm{N}$ et al. A novel coronavirus from patients with pneumonia in China, 2019. N Engl J Med. 2020;

382(8):727-33.

2. Adhikari SP et al. Epidemiology, causes, clinical manifestation and diagnosis, prevention and control of coronavirus disease (COVID-19) during the early outbreak period: a scoping review. Infect Dis Poverty. 2020;9(1):29.

3. World Health Organization (WHO). Coronavirus disease 2019 (COVID-19) Situation Report. 2020. Available at: https://www.who.int/docs/defaultsource/coronaviruse/situationreports/20200331-sitrep-71-covid-19. pdf?sfvrsn=4360e92b_4. Last accessed: 11 June 2020.

4. Cohen J, Kupferschmidt, K. Countries test tactics in 'war' against COVID-19. Science. 2020;367(6484):1287-8.

5. Liang $\mathrm{C}$ et al. Novel coronavirus and orthopaedic surgery early experiences from singapore. J Bone Jt Surg Am. 2020;102(9):745-9.

6. Ashford RU et al. Annotation: the COVID-19 pandemic and clinical orthopaedic and trauma surgery. J Clin Orthop Trauma. 2020;11:504-5.

7. The Guardian. Stay home: Varadkar announces sweeping two-week lockdown. 2020. Available at: https:// www.theguardian.com/world/2020/ mar/27/stay-home-varadkar-urgesirish-in-drastic-lockdown. Last accessed: 11 June 2020.

8. Jordan A. Coronavirus Ireland news: restrictions improve air quality in Dublin and Cork's worst traffic blackspots. 2020. Available at: https://www.irishmirror.ie/all-about/ road-traffic-accidents. Last accessed: 11 June 2020.

9. The Royal College of Surgeons of England. Clinical guide to surgical prioritisation during the coronavirus pandemic. 2020. Available at: https:// www.rcseng.ac.uk/coronavirus/ surgical-prioritisation-guidance/. Last accessed: 11 June 2020.

10. Varghese C, Xu W. Quantifying what could have been - the impact of the Australian and New Zealand governments' response to COVID-19. Infect Dis Heal. 2020; doi: 10.1016/j. idh.2020.05.003.

11. Australian.gov.au. Coronavirus (COVID-19). 2020. Available at: https://www.australia.gov.au/. Last accessed: 11 June 2020.

12. Central Statistics Office. Census 2016 Reports. 2016. Available at: https://www.cso.ie/en/census/ census2016reports/. Last accessed: 28 July 2020.

13. Walker L. 2O19-nCoV acute respiratory disease. Aust Epidemiol Rep. 2020;6:44.

14. Chen $\mathrm{N}$ et al. Epidemiological and clinical characteristics of 99 cases of 2019 novel coronavirus pneumonia in Wuhan, China: a descriptive study. Lancet. 2020;395(10223):507-13.

15. Zhou $\mathrm{F}$ et al. Clinical course and risk factors for mortality of adult inpatients with COVID-19 in Wuhan, China: a retrospective cohort study. Lancet. 2020;395(10229):1054-62.

16. Tande AJ, Patel R. Prosthetic joint infection. Clin Microbiol Rev. 2014;27(2):302-45.

17. Pichard R et al. Impact of the COronaVIrus Disease 2019 lockdown on hand and upper limb emergencies: experience of a referred university trauma hand centre in Paris, France centre in Paris, France. Int Orthop. 2020:1-5.

18. Farley F et al. Avocado-related knife injuries: describing an epidemic of hand injury. Am J Emerg Med. 2019; doi: 10.1016/j.ajem.2019.06.051.
19. Eddleston M, Gunnell D. Preventing suicide through pesticide regulation. Lancet Psych. 2020;7(1):9-11.

20. Holmes $E$ et al. Multidisciplinary research priorities for the COVID-19 pandemic: a call for action for mental health science. Lancet Psych. 2020;7(6):547-60.

21. Markus H, Brainin M. COVID-19 and stroke - a global World Stroke Organization perspective. Int J Stroke. 2020;15(4):361-4.

22. Ducournau F et al. COVID-19: initial experience of an international group of hand surgeons. Hand Surg Rehabil. 2020;39(3):159-66.

23. Hwee $\mathrm{J}$ et al. The impact of coronavirus disease 2019 (COVID-19) on the practice of hand surgery in Singapore. J Hand Surg Am. 2020;45(6):536-41.

24. Facchin F et al. COVID-19: Initial experience of hand surgeons in Northern Italy. Hand Surg Rehabil. 2020; doi: 10.1016/j. hansur.2020.04.007.

25. Leibner $\mathrm{E}$ et al. Emergency department COVID management policies: one institution's experience and lessons learned. Emerg Med Pract. 2020;22(Suppl 5):1.

26. Guo $X$ et al. Survey of COVID-19 disease among orthopaedic surgeons in Wuhan, People's Republic of China. J Bone Jt Surg. 2020;102(10):847-54.

27. Rodrigues-Pinto $\mathrm{R}$ et al. Preparing to perform trauma and orthopaedic surgery on patients with COVID-19. J Bone Jt Surg. 2020;102(11):946-50.

28. Awad M et al. Perioperative considerations in urgent surgical care of suspected and confirmed COVID-19 orthopaedic patients: operating room protocols and recommendations in the current COVID-19 pandemic. J Am Acad Orthop Surg. 2020;28(11):451-63. 
29. Menendez ME et al. Orthopedic surgery post COVID-19: an opportunity for innovation and transformation. J Shoulder Elb Surg. 2020;29(6):1083-6.

30. Grandizio LC et al. Telemedicine in hand and upper-extremity surgery. Hand Surg Am. 2020;45(3):239-42.

31. Mauffrey C, Trompeter A. Lead the way or leave the way: leading a department of orthopedics through the COVID-19 pandemic. Eur J Orthop
Surg Traumatol. 2020:30(4):555-7.

32. Bandyopadhyay $O$ et al. Long-bone fracture detection in digital X-ray images based on digital-geometric techniques. Comput Methods Programs Biomed. 2016;123:2-14 